\title{
TF-IDF Based Contextual Post-Filtering Recommendation Algorithm in Complex Interactive Situations of Online to Offline: An Empirical Study
}

\author{
Cong YIN, Liyi ZHANG, Meng TU, Xuan WEN, Yiran LI
}

\begin{abstract}
O} 2 \mathrm{O}$ accelerates the integration of online and offline, promotes the upgrading of industrial structure and consumption pattern, meanwhile brings the information overload problem. This paper develops a post-context filtering recommendation algorithm based on TF-IDF, which improves the existing algorithms. Combined with contextual association probability and contextual universal importance, a contextual preference prediction model was constructed to adjust the initial score of the traditional recommendation combined with item category preference to generate the final result. The example of the catering industry shows that the proposed algorithm is more effective than the improved algorithm.
\end{abstract}

Keywords: context information; contextual post-filtering recommendation; contextual preference; item category preference; TF-IDF

\section{INTRODUCTION}

At present, the domestic mobile electronic commerce is in full swing, in addition to the related policies, the trend of online and offline convergence is increasing with each passing day. While promoting the consumption pattern and optimizing the industrial structure, the online mode also faces the problem of information overload It is increasingly difficult for users to find the infor matches their needs in the vast amount of services. Especially in $\mathrm{O} 2 \mathrm{O}$ en alize and $\mathrm{d}$
offline interaction is more cont raled. Personalized preference con to sensi when users consume anc decisio How el ctively excavate the prefere and re menornformation that meet eir contextu eeds is be studied and deemed. fecti met s solve the infor atio verload he pe adized preferen is mi through th us tem ary relationship a. is informatio the con $\mathrm{ms}$ to his/her interest is re of mended to the r it t? However, the individualized demand in $\mathrm{O}^{-}$en ironment is not fixed, but has contextual sen Iti ty that is, the personalized preference is differ it in d. ferent contents Previous studies have dem a trate Aat contex ${ }^{\dagger}$ an facto play an important on ation. Herlocker al in tha it is not ep igh to onsider the user score the fac on the proc s 1 experience should be tak nt consideration of 2. 5 , which is the at est resea $h$ literature to $b r$ ine context int the individualized recommenawion. Subsequently, Beitman et al. [6] Yu et al. [5] Gorgoglione et al. [8] and Mallat et al. [9] discussed the influence of contextual factors on user behavior and decision-making mechanism in complex contexts and demonstrated the importance of context to personalized recommendation. Although the related domestic research started relatively late, there are many achievements: Zhuang used Logistic regression model to analyse the user shopping's context factors and found that some contexts have obvious influence on the user's purchase decision [10]. From the four dimensions of physical context, social context, behaviour context and psychological context, Tu Li studied the impulse shopping.
The results show thet the ur dime
ns of context have
shopping, but the influence th tifferent. $\mathrm{P}$ ical text and behaviour conte affect purch social context and p. iro ical co xt influence the unplanned purchase 11]. Hu Cai [1 3], Zhang et al. [14] studied the influence contexulal factors on user demand and preferences om the perspective of user experi Acco ling to the existing research, the contex to a re $\lambda$ on personalize recommendation. is ap puts the context factor the me position a th whe and the item, and d veope the perso a ze commendation based o e nalti-variats rela is ship betwer user, co te ta viem.

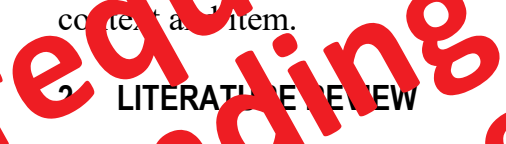

4. an important fa cor to cect user behaviour and cis on-making, the nte information is combined into the persoaliz a nendation $n$ ass, mining the user contextyon $\mathrm{pr}$ fe inces, reco lin information that ment e nueds of the in le current context. This re onmundation mor 1 an effectively analyse the 3 rramic us $r$ ntuxtual preferences, improve recommendâ io quality and user satisfaction. However, tis re re is hent flaws, which are manifested in:

\section{The Data Sparsity Issue in Personalized \\ Recommendation of Fusion Context}

The traditional recommendation almost all faces serious data sparse problem, but the personalization recommendation of the fusion context faces even more serious data sparse problem: on the one hand, the personalized recommendation of fusion context is further deepened in the traditional recommendation, thus the traditional sparse problem still exists. On the other hand, the introduction of context information leads to the transformation of data from traditional user-item twodimensional data to user-context-item multidimensional data, which invisibly expands the data sparsity, because users do not inter-act with the item under some conditions. In the e-commerce environment, the number of users and products is often counted in millions, but the number of 
products commented by users is often limited. The ratio is about $3 \%$, and the possibility of selecting overlapping items between two users is very low [15]. The sparsity of the most studied MovieLen data set is $4.5 \%$, the Bsonomy is $0.35 \%$ and the Delicious is $0.046 \%$ [16]. In the $\mathrm{O} 2 \mathrm{O}$ environment, the relevance degree of the user and the item under most of the context instance conditions is 0 , or the user does not interact with most items under the condition of many associated context instances, that is, the preference degree under the current context is unknown [17]. At this point, the majority of the elements in the high dimensional matrix are empty values. How to generate a relatively precise recommendation using a few non-empty values of the contextual preference data needs to be studied in depth. There are many ways to solve the data sparsity issue in traditional recommendation, such as Wang et al, who combine the similarity of user attributes and item preference into the traditional similarity calculation, and improve the accuracy of nearest neighbour search [18]. Li et al. improve the accuracy of the nearest neighbour search by weighting item category similarity and scoring similarity. This paper combines the item preference degree and item category preference weighting to find the nearest neighbour of the target user so as to reduce the sparsity of the data [19].

\subsection{The Contextual User Preference Extraction} Based on Context Fusion

Contextual user prefence trac $h$ is contextual recomm an. ive iltering recommendation un uses q titat nethod to analyse use rerence, ch is, o tifying preference degree $\&$ numerical ing and calculating it. M men vector pring model ar to the rera cal tel are used to represent us a sice an ition. action techniques nd de nearest 100 gormam, clustering, si - arity cuculation, $[\tau$, 2. c. Sh explicitly gives ther fa tees of som a gie con $t$ instances, calculates th imilarity of on $x+c$ by using hierarchical distance and Jaccard $\delta$ effi ient, and then calculates the user preferene of 1 undimensioncontexts by weighted aggregat $n$. clustering and collaborat ie iltering to model co textural user preferences 1.10 Hot al use ru re ing to calculate Astor Ic ntextual prefe and e andecision tree to ex ct cun at pref repce 24 Bnningen etol use math ma ic. statistics to ns u an overvie is od to calcu contextual user re rences [25]. Com $\mathrm{rcd}$ with the traditional user-item two-dimensional scoring matrix, the contextual user preference extraction of the fusion context faces the dilemma of large amount of data and complex calculation, and the dynamic change of user contextual preference. How to acquire contextual user preferences in a timely and effective manner is worthy of further study.

\section{RESEARCH THEORIES AND METHODS}

\subsection{Theoretical Foundation}

TF-IDF (Term Frequency-Inverse Document Frequency) is a measure of the importance of a word to a document in a corpus. If a word appears frequently in one document and rarely in the others, it means that the word has a good distinguishing ability. This model mainly involves two factors: word frequency and reverse file frequency.

In a given document set, term frequency $(T F)$ refers to the frequency of a word appearing in a document. Considering the length of the document, the same word has a higher frequency in the long document than in the short document. It is necessary to standardize the word frequency (word occurrence frequency divided by the total number of words in the document) to prevent it from biased to the long document. For words in a particular document, their importance can be expressed as:

$T F_{t i}=\frac{n_{t i}}{N}$

where $n_{t i}$ is the freque of tho ord $t$ appearing in document $i$ and $N$ ency of words appearing in
document $D_{t}$.

Iny Do her requ $\mathrm{v}$ is used to measure th rsal im ance of $w$. The more documents it appean the lov the tinguishing ability of the word, the low he impo ce. It is usually obtained from the quotient l rithm between the total number of document and the nu er of documents in which a particular wo app $\mathrm{s}$, and can be expressed as:
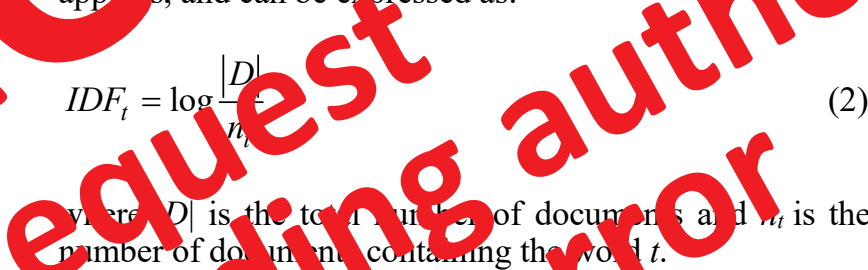

$T F-I D F$ S u ualy obtained y ar tiplying $T F$ value and IDF Il ich indicates th the importance of words IC as with the ino a frequency in the document, (t) ecreases with he crase of the number of documents it appears in. the for ulla can be $\mathrm{pr}$ sed as:

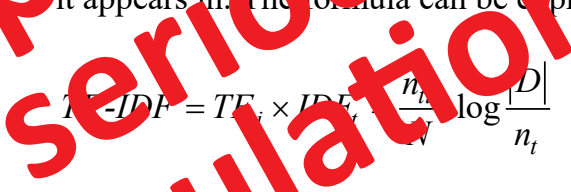

S 0 of the classical algorithms of weight a un, $T F-I D F$ algorithm is popular in the field of (1) formation retrieval and text analysis. Many scholars have extended it to feature selection, domain dictionary construction and user preference modelling. Yan et al. have considered the combination of single word vector and multiple word vectors based on TF-IDF model to calculate the similarity of Weibo information flow and evaluate user interest [26]. According to the item label information selected by users, Zhang et al. build preference prediction models using TF-IDF algorithm and user support degree for items and combine them linearly with the network structure based on recommendation [27].

This paper introduces $T F-I D F$ model into the construction of contextual preference prediction model. The $T F$ value is used to measure the probability of user association with each item in the current context, and the $I D F$ value is used to measure the universal importance of the current context. A contextual preference prediction model is constructed by combining the two models. 


\subsection{TF-IDF Based Post-Context Filtering Recommendation}

According to the user contextual preference, the postcontext filtering recommendation paradigm constructs the preference prediction model and generates the recommendation by adjusting the initial prediction score of the traditional recommendation. In this paper, a $T F-I D F$ based contextual post-filtering recommendation algorithm is proposed according to the characteristics of $\mathrm{O} 2 \mathrm{O}$ and the deficiencies of existing research. The framework of this algorithm is shown in Fig. 1.

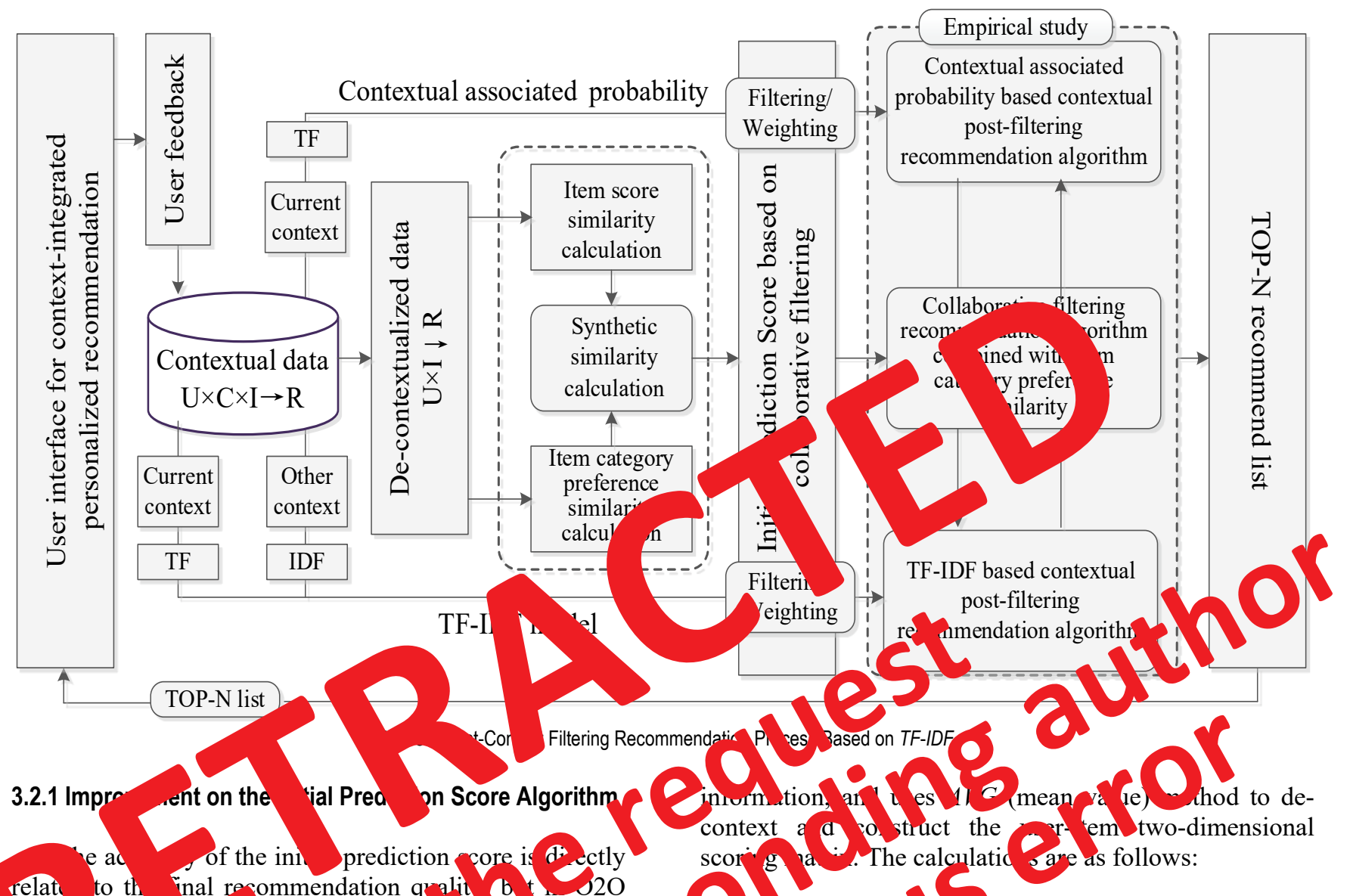

relat to th inal recommendation qualit o t 320 nvi ament,
nat common score item th data sparsity prob em 1. tremery serious, which are ly stricts the co rac of prediction score. At the ne time, in 0 or ario, the user pays attention to the item imal as all as the category of the item, which is minl re eced in the preference for the item categor For cxample, the $c t$ users pay attention to th ategory of fond a d verage (Sichuan cuisine an n oine, etc.). Cor pa with the item, the numb of tem categorie is imiter, and the us common sore on the it $n$ ate ol is much high than the om no score on th $m$ itc aff.

reierence is paper, the sin la y of item category reierence is incorporated into the nearest neighbour search, and the traditional item score similarity and item category preference similarity are combined and weighted to calculate the synthetic similarity to search for nearest neighbour user, predict the user initial score of the item and improve the accuracy of the prediction score.

Item score similarity calculation. The consumption behaviour in $\mathrm{O} 2 \mathrm{O}$ environment mostly belongs to regular and high frequency consumption behaviour. Users may have multiple scores for the same item in different contexts or the same context. The similarity calculation of item scoring needs to construct the user-context-item multidimensional scoring matrix which integrates context
I $a_{a}$ core $_{d}(u, i d)$ t es mof user $u$ score on item P.de the context $c$ nd or a , and $n_{d}$ is the frequency of scorif o $\mathbb{1}^{\mathrm{t}} \mathrm{n}$. nder the context condition $d$. The user-item tu - msional scoring matrix can be consuc ed y alculation and the similarity of user score n e garulated by using Pearson Correlation Similarity. Th sunilarity between $a$ and $u$ can be calculated as follows

$$
\operatorname{Sim}_{R}(a, u)=\frac{\sum_{i \in I_{a u}}\left(R_{a, i}-\bar{R}_{a}\right)\left(R_{u, i}-\bar{R}_{u}\right)}{\sqrt{\sum_{i \in I_{a u}}\left(R_{a, i}-\bar{R}_{a}\right)^{2}} \sqrt{\sum_{i \in I_{a u}}\left(R_{u, i}-\bar{R}_{u}\right)^{2}}}
$$

where $a$ is the target user, $u$ is the other user, $I_{a u}$ represents a set of items commented by user $a$ and $u$.

Item category preference similarity calculation. For a given contextual data set $C D$ in the $\mathrm{O} 2 \mathrm{O}$ environment, the set of items being commented on is $I, I=\left\{I_{1}, I_{2}, \ldots, I_{j}\right\}$, The items generated by clustering or categorization belong to a collection of categories $V, V=\left\{V_{1}, V_{2}, \ldots, V_{l}\right\}$, The items contained within the categories are as similar as 
possible, and the items in each category are as different as possible, and the items are satisfied with the requirements of $I=V_{1} \cup V_{2} \cup \ldots \cup V_{l}, V_{i} \cap V_{j}=,(1 \leq i \leq l, 1 \leq j \leq l)$, According to the user score on the item and the corresponding scoring frequency, the item category preference degree is calculated, and the user-item category preference matrix is constructed. The calculation of item category preference [26] is as follows:

$P_{u, v_{i}}=\frac{\operatorname{allScore}\left(u, v_{i}\right)}{\operatorname{allScore}(u, V)}$

where allScore $\left(u, v_{i}\right)$ is the sum of the item scores included in category by user $u$, and allScore $(u, V)$ is the sum of item scores in all item categories by user $u$. The Pearson Correlation Similarity is used to calculate the item category preference similarity in the constructed user-item category preference matrix. The formula is as follows:

$$
\operatorname{Sim}_{V}(a, u)=\frac{\sum_{v \in V_{a u}}\left(P_{a, v}-\bar{P}_{a}\right)\left(P_{u, v}-\bar{P}_{u}\right)}{\sqrt{\sum_{v \in V_{a u}}\left(P_{a, v}-\bar{P}_{a}\right)^{2}} \sqrt{\sum_{v \in V_{a u}}\left(P_{u, v}-\bar{P}_{u}\right)^{2}}}
$$

where $V_{a u}$ represents a set of item categories by user $a$ and $u$.

Synthetic similarity lo tion he va score similarity Sir a) an forence similarity $\operatorname{Sim} V(a, \operatorname{Sim}(a, u$ t calcu $n$ is

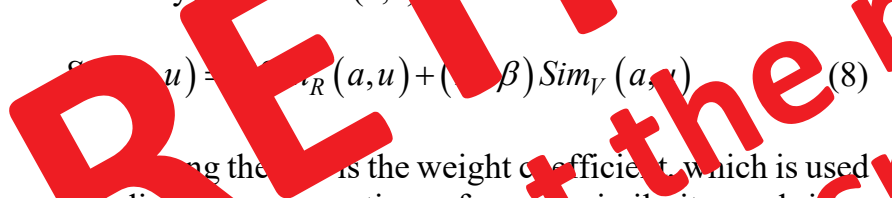
(8)

\section{2} terr. In a fiv poi coring system, a score of 3-5 points important to determine the

Improved i itiol ediction sco lation. Calculate the it $n$ sis Similarity $\operatorname{Sim}(4)$, and select the neigh our or he op K as the n arest one set to predic the tan ther a's tradition $1 \mathrm{Co}$ muded incial o for each ter The calculati ny a is as follow. $P_{a, i}=\bar{R}_{a}+\frac{\sum_{u \in U} \operatorname{sim}(a, u) \cdot\left(R_{u, i}-\bar{R}_{u}\right)}{\sum_{u \in U}|\operatorname{sim}(a, u)|}$

where the $\bar{R}_{u}=\left(1 /\left|I_{u}\right| \sum_{u \in I_{u}} R_{u, i}\right)$ is user $u$ 's average score on items, and $I_{u}=\left\{i \in I \mid R_{u, i} \neq \varnothing\right\}, U$ is a set of all items. and the imp an e the current of ite in each item.

$T p$ obinty of the $s c$ a sociation with each itam $t$ current co cext. 1 asually measured by the ati or the target iss 's po itive feedback frequency to the

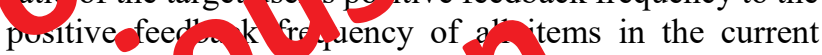
context $5 \mathrm{he}$ hig er the cont a a ciation probability is the rea $r$ the userpr en nce for the item in the current atext. Positive faed ack is the user preference for this indicate "I e puoftive feedback, expressed by $t=1$ ). The rob coll contextual association of target user a to item

\subsubsection{Construction of Contextual Preference Prediction Model}

Contextual preference prediction model directly affects the accuracy of filtering recommendations and user prediction model is the key to this context and the associated probability of construct the contextual preference prediction model, which can excavate the user preference for each purpose in the current context, but the method treats the importance of the current context in each item equally, and the importance of the same context in different items is . Generally speaking, the more contexts an item addition, the lower its contextual importance is, in item, the higher its cor ortance, that is, the contextual importance inverse proportional to the number of con invol in the 1

In orer effectivel neasy the importance of

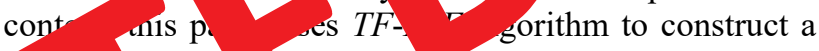
ali mode f contextuar preference, $T F$ is used to in the ce nt contuxt, and $I D F$ is used to calculate the importanc $\int$ the current context in each item. If th has higher probability of associating an it t $\mathrm{e}$ hit context, and importance of the ant $n$ ontext In the item is hig er th the user is m re oglmed to the item in the c r n $\Omega$ ntext. Thu $\rightarrow$ ul be seen that the predic on nu 1 of contextual is erence baser on $T F$ I $\Gamma$ g im mainlyin o es two aspects p bability If $\Gamma$ g item in te ur nt context $i$ c $\mathrm{rr}$ nt context $\mathrm{c}$ can be calculated as:

$T F_{a, c, i}=\frac{\operatorname{count}\{t=1 \mid i, C=c\}}{\operatorname{count}\{t=1 \mid I, C=c\}}$

where count $\{t=1 \mid i, C=c\}$ is the total number of positive feedbacks of user $a$ to the item $i$ under the current context $c$, count $\{t=1 \mid I, C=c\}$ is the total number of positive feedbacks of user $a$ to all items $I$ under the current context c.

The importance of current context in each item. In order to measure it, this paper uses the $I D F$ method to measure the universal importance of the current context in each item. The more contexts the item involves, the more disperse the preferences of each context to the item, the lower the importance. The universal importance of the current context $c$ in item $I$ can be obtained by dividing the total number of contexts $N_{I}$ involved in all items by the satisfaction. How to construct an effective contextual 
number of contexts $N_{i}$ that item $i$ relates to, and then taking the resulting quotient logarithm, that is:

$I D F_{c, i}=\log \frac{N_{I}}{N_{i}}$

Contextual preference calculation based on $T F$ IDF algorithm. According to the $T F-I D F$ algorithm, the calculation formula of the target user contextual preference for all items in the current context is as follows:

$$
P(a, c, i)=T F_{a, c, i} \cdot I D F_{c, i}=\frac{\operatorname{count}\{t=1 \mid i, C=c\}}{\operatorname{count}\{t=1 \mid I, C=c\}} \cdot \log \frac{N_{I}}{N_{i}}
$$

The above formula can be expressed as the degree the user preference for an item in the current context increases with the increase of the user preference frequency for the item in the current context, but decreases with the increase of contexts in which the user chooses the item. To make it easy to quantify, the TFC weight algorithm [29] is used to normalize the calculated values of formula (12), and the contextual preference values with a value range of $[0,1]$ are obtained.

\subsubsection{Generation of Contextual Post-Filtering Recommendation}

That is to use the contextual pal the traditional recommendation ial sc e to final score. There are to foth of direct filtering and scor ction. Iric yo es have shown that of two a stment methods is superior e other [30]. s paper mbines the two to adiuct the $\mathrm{lit}$. 1 recom nded prediction $\mathrm{sc}$. 0 en prod is as follows.

D ct fil no me items that ha his sures but D et fil no me items that ha his sures but

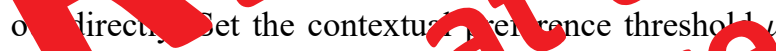
ad ce, when $P(a, c, i)<\mu$, ch $n$ s the original pr action score of the traditional recommendation to th is $\Gamma_{a, c, I}=$ 0 .

Score correction. The rema ing it hs weighted th initial prediction score $P \cap$ nd th ontextual feren value $P(a, c, i)$, that is se a's final sc $c t$ e item in current ont $\mathrm{c}$ \& $\Lambda_{c, i}$, TOP-N momn atation was generated the ca onlation is sho un follows:

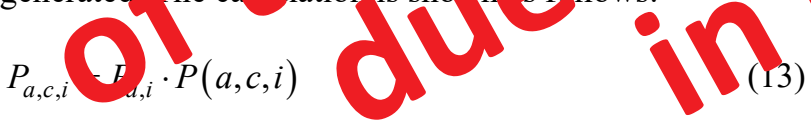

\section{EMPIRICAL ANALYSIS}

\subsection{Experimental Data}

This paper takes Wuhan catering industry of Dianping.com as the experimental object, using crawler tools to grab eight data segments including store information, user consumption information and comment information, including user name, consumption time, store name, address, store style, user rating and comment content etc. A total of 132 users, 60142 review records and 3295 stores are involved.

\subsection{Associated Context Information Acquisition}

The context acquisition method includes explicit acquisition and implicit acquisition; explicit acquisition is the most accurate but difficult to most valid contexts. This paper uses implicit acquisition method to associate relevant context information in food and beverage $\mathrm{O} 2 \mathrm{O}$ environment:

Location. According to the Wuhan geographical location of the stores, including seven examples: Wuchang, Hongshan, Qingshan, Hanyang, Jiangan, Jianghan, Qiankou;

DayType. According to the user consumption date associated to the user status and combined with the general office of the State Council holiday adjustment arrangements to adjust the acquisition including three examples: working days, weekends and holidays.

Weather. According consumption date associated with the $\mathrm{w}$ rer, in ing five examples: overcast, sunny, sno nd clou

Compan Extrace pers al pronouns for associa fro bo co ermation, including fo nples: a e, friend, $\mathrm{A}$,er and family.

En ion. Th not 1 polarity of user comments is calculate obtain emotional context, including three examples. sitive, negative and neutral.

In conc.usions, the context dimension and its ex mp in $O$ environment $\mathrm{r}$ shown in Tab. 1

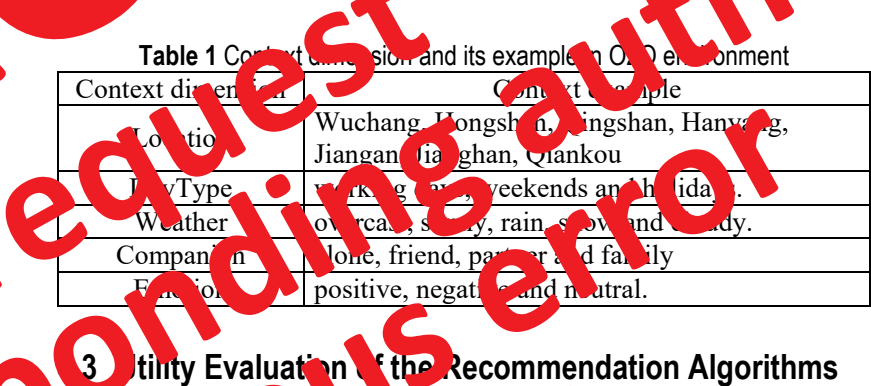

3) tinty Evaluat' nn the Recommendation Algorithms

In or en verify th 1. ity of the proposed re enation alg $n$, th paper uses the Mean A solute Errer $(M-y)$, Tre sion and Recall to determinate le optimal w igh $c$ efncient and the optimal contextual preferen th es ora [31], and to evaluate the utility of the F $I F$ b i post-filtration recommendation algorithm.

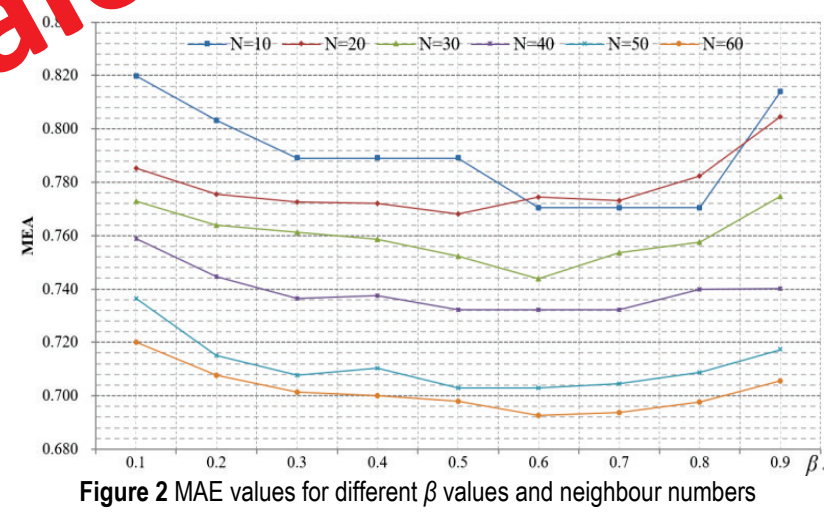

\subsubsection{The Optimal Weight Coefficient Determination}

During initial prediction scoring, the traditional score similarity and the item category similarity are weighted. 
The weight coefficient is closely related to the accuracy of the prediction score. In this paper, the weight coefficient $\beta$ is increased from 0.1 to 0.9 at 0.1 intervals, the nearest neighbour number increases from 10 to 60 at 10 intervals, and the corresponding $M A E$ value is calculated as shown in Fig. 2. It can be seen from the graph that the average absolute error $M A E$ value is the smallest, that is, the best weight coefficient occurs when $\beta=0.6$.

\subsubsection{The Optimal Contextual Preference Threshold Determination}

In direct filtering, the contextual preference threshold $\mu$ is too large, which results in the items related to the current context being filtered out, and the items that are irrelevant to the current context being mixed into the recommendation list, both of which will lead to the poor quality of the recommendation.

To explore the most suitable threshold, the contextual preference threshold is set from 0.9 to 0.1 , decreasing by 0.1 , and the initial prediction score is adjusted by direct filtering and score correction. When the threshold $\eta$ is 0.9 , 0.8 and 0.7 , respectively, the number of items with a score which is not 0 is too small to calculate the corresponding evaluation index value, so the three thresholds are abandoned. The Precision and Recall statistics are shown in Fig. 3 and Fig. 4. It can be seen from the dir am that when the threshold $\eta=0.2$, the correspondin and Recall values are better than the other preference thresholds, so $\eta=0.2$

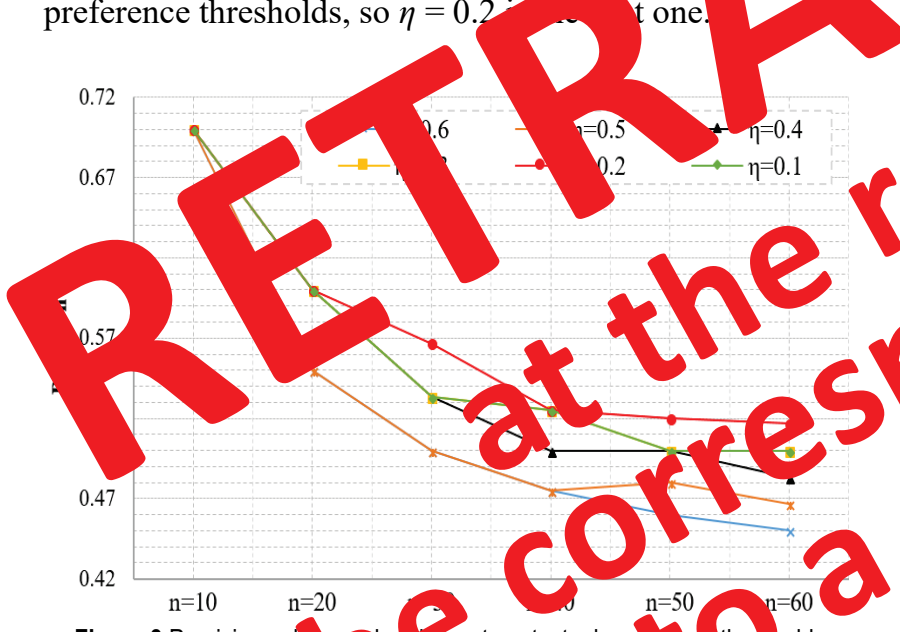

Figure 3 Precision valu s erent contextual pre the thre holds
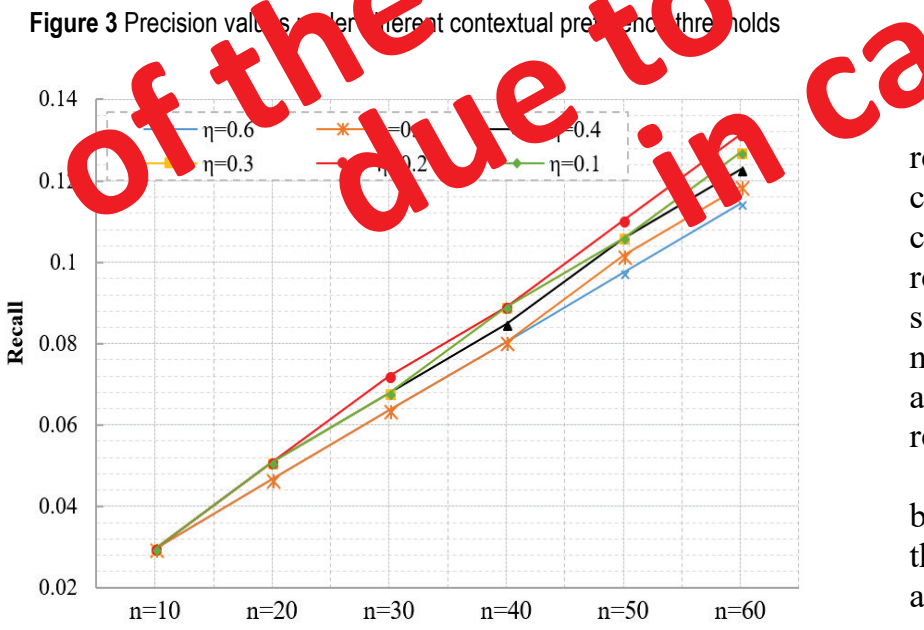

Figure 4 Recall values under different contextual preference thresholds

\subsubsection{The TF-IDF Based Contextual Post-Filtering Recommendation Algorithm's Utility Evaluation}

To verify the validity of the $T F-I D F$ based contextual post-filtering recommendation algorithm ( $T F$ $I D F$ based_CPF), this paper compares it with the collaborative filtering recommendation algorithm combined with item category preference similarity (ICPS_combined_CF) [32] and the contextual associated probability based contextual post-filtering recommendation algorithm (CAP_based_CPF) [30]. Related index statistics are shown in Fig. 5 and Fig. 6.

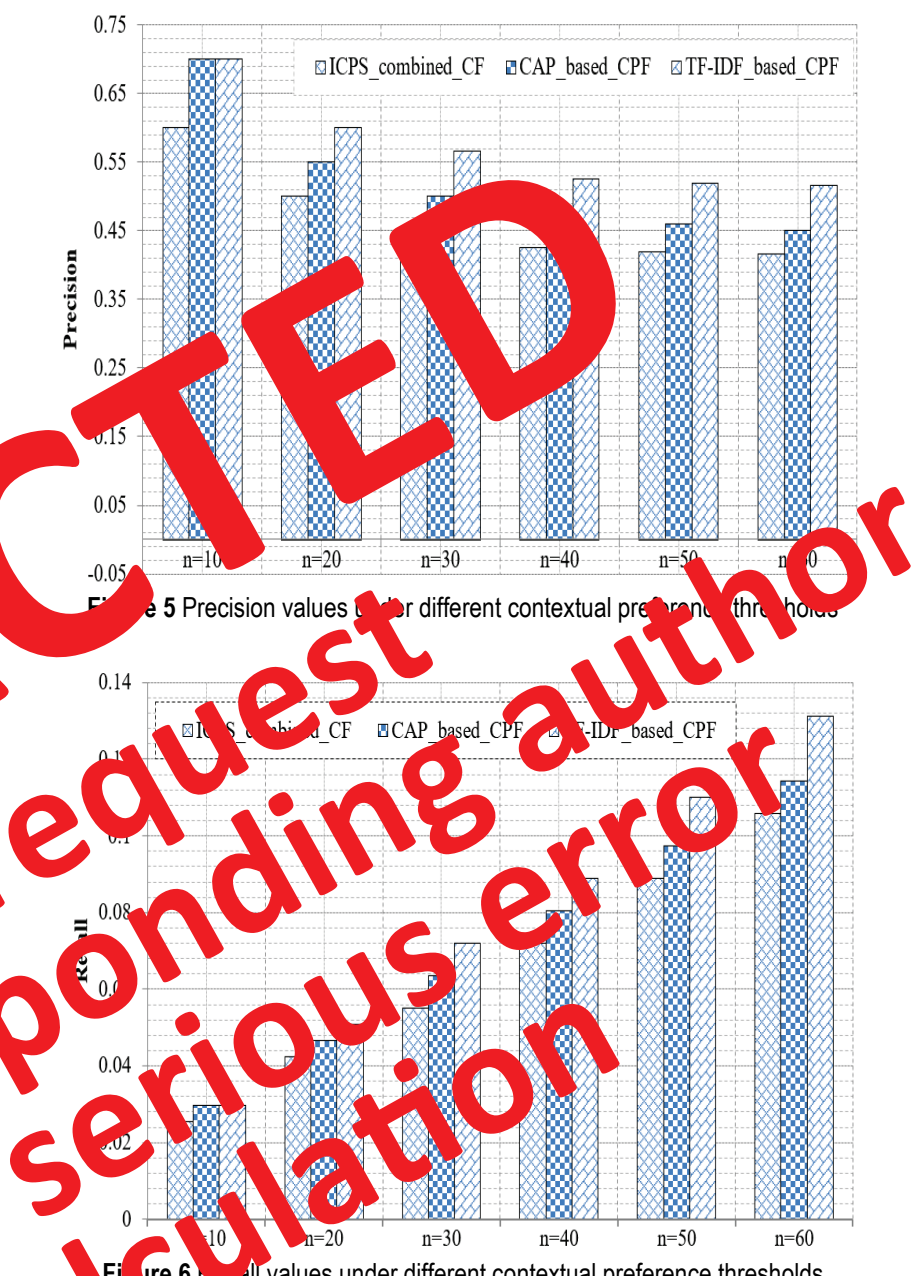

$\mathrm{Fi}$ Ire 6

As can be seen from the graph:

The TF-IDF based contextual post-filtering recommendation algorithm is obviously superior to the collaborative filtering recommendation algorithm combined with item category preference similarity, the reason is: The users' personal needs have contextual sensitivity and change with the context change, which is not fixed, and it shows that the context information plays an important role in the process of personalized recommendation.

Compared with the contextual associated probabilitybased contextual post-filtering recommendation algorithm, the $T F-I D F$ based contextual postfiltering recommendation algorithm has advantages in Precision and Recall values, and the advantages become more obvious as the recommended item number increases. This shows that the proposed algorithm can effectively improve the contextual 
preference extraction quality and then improve the recommendation quality by further analysing the item's context importance based on considering the contextual association probability. It is proved that the algorithm is effective.

\section{CONCLUSIONS}

This paper studied the information overload problem in $\mathrm{O} 2 \mathrm{O}$ environment, improved the contextual associated probability-based contextual post-filtering recommendation algorithm, constructed contextual preference prediction model based on contextual association probability and contextual universal importance to extract the contextual preference degree. Based on this, the direct filtering and score correction were applied to the initial prediction score of collaborative filtering recommendation combined with item category preference to generate the recommendation. The utility evaluation verifies that the best weight coefficient is 0.6 and the optimal contextual preference threshold is 0.2 . Furthermore, the proposed $T F-I D F$ based contextual postfiltering recommendation algorithm is obviously superior to other algorithms, and with the increase of the recommended items number, the advantages are more obvious.

\section{Acknowledgment}

This work is supported Foundation of Chongqino

REFERENCF

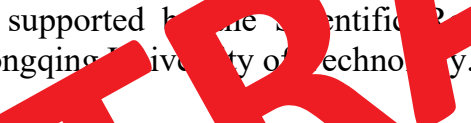

Smartphones. IEEE International Conference on Mobile Cloud Computing. IEEE Computer Society, 68-75. https://doi.org/10.1109/MobileCloud.2014.26

[8] Gorgoglione, M., Palmisano, C., \& Tuzhilin, A. (2006). Personalization in Context: Does Context Matter When Building Personalized Customer Models? Proceedings of the $6^{\text {th }}$ IEEE International Conference on Data Mining (ICDM 2006), 18-22 December 2006, Hong Kong, China. IEEE. https://doi.org/10.1109//CDM.2006.125

[9] Mallat, N., Rossi, M., Tuunainen, V. K., \& Rni, A. (2009). The impact of use context on mobile services acceptance: the case of mobile ticketing. Information \& Management, 46(3), 190-195. https://doi.org/10.1016/j.im.2008.11.008

[10] Gui-Junzhou, Z. (2004). Impact of situational factors on shoppers' buying decision: an initial study. Application of Statistics \& Management, 23(4), 7-13.

[11] Tu Li. (2010). The Influence of Situational Factors on Impulse Buying Behavior. Hubei University, 2010.

[12] Mu H. Hu \& Shu Q. Cai. (2010) Personalized recommendation method $\mathrm{V}$ coupled situation with Extended Research. ary ana rmation Service, 371376.

[13] Han Li. 4) The rent $S$ of Context-aware Rese h. upute and rmat Technology, 22(6), 4Zhao, lin Li \&
an s of onl Zhang. (2019). A metaof ed mic dev pment. International Journal of Medical Inform s, 127(7), 68-79. https://do org/10.1016/j.jimedinf.2019.04.015

[15 Ingseth, H. \& Nilsen, T. D. (2012). collaborative filtarin International Joux of o pr ximate Reasoning $53(4,166$. https://doi. reno. 10.0/j.jijar.2011.1 n 2

[16] Lii Q. H., cang, S., \& Thang C. (2016). The nfluence (1) mation cascac s in online purcha he viors of 1] Borch A., Urlocker, Konstan, J., \& Reidl, J. (1998). [1] Borch A., Urlocker, Konstan, J., \& Reidl, J. Research 6(, 53-8, $\mathrm{https} / / \mathrm{d}$ or 10 .007/s10660-0 o 2. - 0

17] I A Lhang, X. V., Zh g. Y. Y., \& Zhao, Y. (2018).

Th ineraction effe $\mathrm{f}$ information cascades, word of houth and re om endion systems on online reading behamior An n cal investiget on Electronic Commerce Res a h -2

[101 fy, Zhu, Y, Li D., Ch 1, W., \& Wang, Y. (2016). Join. User Attributa nnd tenc Category in Factor Models for Kating PI tivio In Mational Conference on Database Systems or 4d iced Applications. Springer International p $\rightarrow$ hl hin hr.ps://doi.org/10.1007/978-3-319-32025-0_18

1) a-Xuu, L. I., Ming-Liang, X., \& Xue-Bin, Z. (2010). Conaborative filtering recommendation algorithm using item category information. Journal of Chongqing University of Posts and Telecommunications (Natural Science Edition), 22(6), 823-827.

[20] Stefanidis, K., Pitoura, E., \& Vassiliadis, P. (2007). Adding Context to Preferences. Data Engineering, ICDE 2007. IEEE $23^{\text {rd }}$ International Conference on. IEEE. 846-855. https://doi.org/10.1109//CDE.2007.367930

[21] Stefanidis, K. \& Pitoura, E. (2008). Fast contextual preference scoring of database tuples. In: Proc. of the EDBT, IEEE Computer Society, 344-355. https://doi.org/10.1145/1353343.1353387

[22] Shin, D., Lee, J. W., Yeon, J., \& Lee, S. G. (2009). ContextAware Recommendation by Aggregating User Context. 2009 IEEE Conference on Commerce and Enterprise Computing, CEC 2009, Vienna, Austria, July 20-23. 423430. https://doi.org/10.1109/CEC.2009.38

[23] Jrad, Z., Aufaure, M. A., \& Hadjouni, M. (2007). A Contextual User Model for Web Personalization.

[7] Otebolaku, A. M. \& Andrade, M. T. (2014). Supporting Context-Aware Cloud-Based Media Recommendations fo 
International Conference on Web Information Systems Engineering. Springer-Verlag, 350-361.

https://doi.org/10.1007/978-3-540-77010-7_33

[24] Hong, J. Y., Suh, E. H., Kim, J., \& Kim, S. Y. (2009). Context-Aware system for proactive personalized service based on context history. Expert Systems with Applications, 36(4), 7448-7457. https://doi.org/10.1016/j.eswa.2008.09.002

[25] Bunningen, A. H. V., Fokkinga, M. M., Apers, P. M. G., \& Feng, L. (2007). Ranking Query Results using ContextAware Preferences. IEEE International Conference on Data Engineering Workshop. IEEE. 269-276. https://doi.org/10.1109/ICDEW.2007.4401003

[26] Yan Guang-Hui, Chen Yong, Zhao Hong-yun, et al. (2014) Personalized tweet recommendation. Computer Engineering and Design, 35(6), 2013-2016.

[27] Xinmeng, Z., Shengyi, J., Xia, L. I., \& Qiansheng, Z. (2015). Hybrid recommendation algorithm based on network and tag. Computer Engineering and Applications, 51(1), 119124.

[28] Wu, Y. \& Ma, J. (2014). A genre-based hybrid collaborative filtering algorithm. Journal of Computational Information Systems, 10(22), 9831-9838.

[29] Chu Leilei, Chang Wenbo, \& Li Qin. (2012). Improvement of Feature Weight Formula in Text Clustering. Chinese Journal of Engineering Mathematics, 29(4), 523-528.

[30] Panniello, U., Tuzhilin, A., Gorgoglione, M., Palmisano, C., \& Pedone, A. (2009). Experimental comparison of pre- vs. post-filtering approaches in context-aware recommender systems. Proceedings of the 2009 ACM Conference on Recommender Systems, RecSys 2009, New York, NY, USA October 23-25, 265-268. https://doi.org/10.1145/1639714.1639764

[31] Ye, Y., Zhao, Y., Shang, J., \& Zhang, L. Y. (20 IT framework for identifying hi ${ }^{1}$ big data analytics. Internati Management, 47(4), 65 https://doi.org/10.10" omgt.20

[32] Leng Yajun $\mu$,ing, Zhang J ng. (2,). Improved item-b ollaborative tering at thm combined with class Eerence inform n. Appucation Research of

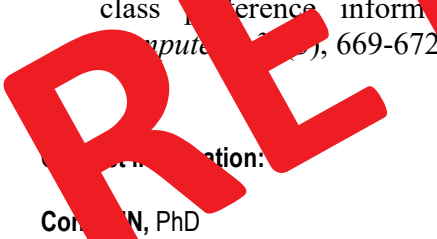

No. 69 Hongguang Avenue, Banan District, Chongqina cyin@cqut.edu.cn

Liyi ZHANG, Professor (Corresponding author)

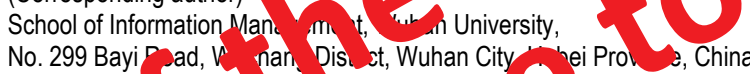
lyzhang@who u.cn

Meng

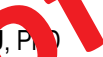

Chonga ellectual Property Sc ol hongqing University of Tt hn Logy,

No. 69 Hongguang Avenue, Banan boutrict, Chongqing, China

amytwe0214@hotmail.com

\section{Xuan WEN, PhD}

School of Information Management, Wuhan University,

No. 299 Bayi Road, Wuchang District, Wuhan City, Hubei Province, China

Xuan_W113@163.com

\section{Yiran $\mathrm{LI}, \mathrm{PhD}$}

School of Information Management, Wuhan University,

No. 299 Bayi Road, Wuchang District, Wuhan City, Hubei Province, China

yiran.li@whu.edu.cn 


\section{apply for retraction one paper}

张李义<lyzhang@whu.edu.cn>

29. prosinca 2019. u 12:43

Prima: tehnvj@sfsb.hr

Dear Prof. Dr. Milan Kljajin, In an article published in your journal, we found serious data errors, the calculation results are inconsistent with the published results, so we apply for retraction. Please help us to retract the article. The authors are very sorry for the trouble caused by our mistake.

\section{The article information:}

Title: TF-IDF Based Contextual Post-Filtering

Recommendation Algorithm in Complex Interactive

Situations of Online to Offline: An Empirical Study,

Tehnički vjesnik 26, 6(2019), 1529-1536

Authors: Cong YIN, Liyi ZHANG,

Meng TU, Xuan WEN, Yiran LI

sincerely,

张李义

Dr. Liyi Zhang

professor, Department of Electronic Commerce

School of Information management

Wuhan university,P.R. China,430072 